\title{
Assessing the hazard from geomagnetically induced currents to the entire high-voltage power network in Spain
}

\author{
Joan Miquel Torta ${ }^{*}$, Santiago Marsal ${ }^{1}$ and Marta Quintana ${ }^{2}$
}

\begin{abstract}
After the good results obtained from an assessment of geomagnetically induced currents (GICs) in a relatively small subset of the Spanish power transmission network, we now present the first attempt to assess vulnerability across the entire Spanish system. At this stage, we have only included the power grid at the voltage level of $400 \mathrm{kV}$, which contains 173 substations along with their corresponding single or multiple transformers and almost 300 transmission lines; this type of analysis could be extended to include the 220-kV grid, and even the 110-kV lines, if more detailed information becomes available. The geoelectric field that drives the GICs can be derived with the assumption of plane wave geomagnetic variations and a homogeneous or layered conductivity structure. To assess the maximum expected GICs in each transformer as a consequence of extreme geomagnetic storms, a post-event analysis of data from the Ebre Geomagnetic Observatory (EBR) during the 2003 Halloween storm was performed, although other episodes coincident with very abrupt storm onsets, which have proven to be more hazardous at these mid-latitudes, were analyzed as well. Preferred geomagnetic/geoelectric field directions in which the maximum GICs occur are automatically given from the grid model. In addition, EBR digital geomagnetic data were used to infer statistical occurrence probability values and derive the GIC risk at 100-year or 200-year return period scenarios. Comparisons with GIC measurements at one of the transformers allowed us to evaluate the model uncertainties.
\end{abstract}

Keywords: Geomagnetically induced currents; GIC; Space weather; Natural hazards; Spain

\section{Background}

Solar-terrestrial physics is a discipline with a long tradition, and many observatories and research groups have been dedicated to this subject for a long time. However, as modern society has become more dependent on technological systems and therefore vulnerable to disturbances in the upper atmosphere and in the interplanetary space near Earth, the focus of this discipline evolved in new and important directions, and it has been renamed as space weather (e.g., Song et al. 2001). Space weather covers the study of the conditions in space that could affect human activity. Phenomena originating in the Sun that can affect human activity include explosive events such as flares and coronal mass ejections. These produce large quantities of

\footnotetext{
*Correspondence: jmtorta@obsebre.es

'Observatori de l'Ebre, (OE), CSIC, Universitat Ramon Llull, Horta Alta 38, Roquetes 43520, Spain

Full list of author information is available at the end of the article
}

energetic particles and solar wind disturbances, which travel through the interplanetary medium and affect the terrestrial environment. This solar activity is cyclical (solar cycle) and reaches a maximum approximately every 11 years.

The event that sparked global interest in this discipline, beyond academia, was the collapse of the power grid in northeastern Canada due to the great geomagnetic storm of 13 March 1989; this event left millions of people without power for $9 \mathrm{~h}$ (Bolduc 2002). In October-November 2003, during the declining phase of the next solar cycle, a series of events known as the Halloween storm occurred, and as a result, some new problems in electrical transmission networks were encountered (e.g., there was a blackout in the city of Malmö in southern Sweden (Pulkkinen et al. 2005; Wik et al. 2009)) and several space missions and satellites experienced numerous anomalies (Royal Academy of Engineering 2013). Motivated by the events 
of 2003, the Committee on Solar and Space Physics (CSSP) of the U.S. National Research Council (NRC) began to consider the need to systematically assess the social and economic impacts of space weather. To establish objectives for this research, the CSSP organized a workshop in Washington, D.C. during May 2008. The conclusions obtained as a result of that meeting can be found in the workshop report (U.S. National Research Council 2008). One of the most obvious conclusions revealed by the historical records is that past space weather events, such as the Carrington event in 1859 (Cliver and Dietrich 2013) and the great geomagnetic storm of May 1921 (Kappenman 2006), have been as much or even more severe than some of the more recent ones; this suggests that such extreme events, although rare, are likely to happen again sometime in the future. As stated in the workshop report, according to a study by the Metatech Corporation, today the occurrence of an event like the 1921 storm would result in large-scale blackouts affecting over 130 million people and would put more than 350 transformers at risk of permanent damage.

Geomagnetic storms caused by disturbances in solar conditions can affect the terrestrial environment including the magnetosphere, ionosphere, and magnetosphere-ionosphere interaction region; such effects are detectable at the surface of the Earth (e.g., Blanch et al. 2013). The highly conductive nature of the ionosphere produces significant current systems in this region, such as the equatorial and auroral electrojets. During disturbed conditions, there is a significant increase in these currents (the auroral electrojet can measure on the order of millions of amps). As these are time-varying, intense induced electric currents appear occasionally in conductors at the Earth's surface. The European Space Agency (ESA) launched the Swarm mission in November 2013 to better understand the magnetic field around Earth. This mission provides opportunities for near real-time in situ monitoring of the ionosphere and upper atmosphere (Stolle et al. 2013).

The induced electric currents generated by geomagnetic storms in ground-based technical networks are called geomagnetically induced currents (GICs). Their frequencies are typically in the millihertz range, and thus, they can be considered quasi-direct currents (quasi-dc). These currents can disrupt and damage our technological systems, e.g., by affecting transformers of high-voltage networks in the case of power transmission systems. Specifically, transformer cores can saturate under the effect of GICs by operating in the saturation region of the magnetization curve $(B-H)$ of the steel core (Molinski 2002; Kappenman 2007). When a large number of transformers experience GIC saturation, the demand for reactive power and the harmonics of the system voltage are significantly increased. Reactive power demands of this magnitude can cause serious changes in the system voltage. At the same time, the change in the size of the ferromagnetic material (magnetostriction) between the saturated and unsaturated states at twice the power frequency produces heat, noise, and mechanical vibration damage (Koen and Gaunt 2002). The transformers may also suffer premature aging due to the effects of GICs. This aging can be detected by measuring the concentration of gas in the transformer oil. The harmful effects of magnetic storms on power grids have been observed both at high latitudes and at low and middle latitudes. Damage has even occurred in electrical networks at latitudes lower than Spain, such as in the case of South Africa during the Halloween storm of 2003 (Gaunt and Coetzee 2007). This event was related to the onset of damage in a $700 \mathrm{MVA}$ transformer and in several smaller power transformers. It has been shown (Torta et al. 2012) that during that event, the amplitude of the variations of the horizontal geomagnetic field and its time derivative $\left(d B_{H} / d t\right)$, which is more important for geoelectric field generation, were significantly higher at the Ebre Observatory in Spain than in Hermanus, South Africa. It is therefore prudent that we assess the effects of magnetic storms on the Spanish power network.

\section{Methods}

Modeling efforts for calculating GICs in ground-based technological systems require a determination of the electric field that occurs in connection with a magnetic storm at the Earth's surface and a calculation of the resulting GIC (see Pirjola 2002). The practical calculation includes both a geophysical and an engineering problem because the surface geoelectric field is determined from geomagnetic recordings or from ionospheric-magnetospheric current data (if available) as the first step, and then the GIC flow is calculated under the given geoelectric field using the known topology and resistances of a conductor system.

For the determination of the geoelectric field, we have different possibilities. Going from the most sophisticated to the simplest one, one can choose exact formulas for the calculation of magnetic and electric fields at the Earth's surface due to an auroral electrojet, which include contributions from field aligned currents (e.g., Pirjola and Häkkinen 1991). However, the resulting formulas are complicated and unsuited to real-time calculations. On the basis of the experience obtained by this study, we attempt to use a practical procedure for the real-time calculation of GICs, which would allow organizations to find the best way of configuring the power system to mitigate their impact as fast as possible.

Considering the real-time calculation, we used approximate solutions. One of them is called the Complex Image Method (CIM) (e.g., Boteler and Pirjola 1998), which is based on the 'method of images' in which the Earth is replaced by an image of the inducing source at a 
complex depth. For a source at height $h$ above the Earth's surface, the complex depth is $h+2 p$, where $p$ is the complex skin depth related to the surface impedance, $Z$ (Wait 1981; Boteler and Pirjola 1998):

$$
p(\omega)=\frac{Z(\omega)}{i \omega \mu_{0}}
$$

Here $i, \omega$, and $\mu_{0}$ are the imaginary unit, the angular frequency, and the vacuum permeability, respectively. Both the complex skin depth and the surface impedance are defined in the frequency domain. As a first approximation to the auroral electrojet, Boteler and Pirjola (1998) derived algebraic expressions for the magnetic and electric fields at Earth's surface due to an infinite line current above the Earth using CIM. They considered two different conductivity models representative of Québec and British Columbia, which are resistive and conductive, respectively. It has been demonstrated that CIM gives a very good agreement with the exact methodology. Although it is a convenient tool for theoretical modeling purposes, it is not the most optimal choice for more operational applications because it was simply derived for a line current above the Earth. However, Pirjola and Viljanen (1991) show that the applicability of CIM is not restricted to the line current case as they extended the use of CIM to a more complicated ionosphericmagnetospheric current system. Pirjola et al. (2000) also show the successful use of CIM for a westwardtraveling-surge (WTS) event.

Since the true 3-D ionospheric current system cannot be determined by using ground magnetometer data only, the method of Spherical Elementary Current Systems (SECS) (Amm 1997) can be used to introduce a horizontal equivalent current system, which produces the same magnetic and electric field at the Earth's surface. This method is based on the fact that geomagnetic variations at the Earth's surface can be explained by a horizontal divergence-free current system at the ionospheric level. The amplitudes of the elementary current systems are determined by the inversion technique, by fitting the modeled horizontal field to the measured one, and once the currents are determined, obtaining the electric field at the Earth's surface due to one element is straightforward. Inversion is usually resolved by the singular value decomposition technique because the system is typically underdetermined. Data for just three magnetic observatories have even been reported to have been used in the interpolation process to infer the equivalent ionospheric currents (Caraballo et al. 2013).

The CIM and the SECS technique are commonly used to determine the geoelectric field at high geomagnetic latitudes. Pulkkinen et al. (2003) presented a calculation using a combination of both techniques. At mid-latitude regions, however, the source field is rather uniform, and if we happen to have geomagnetic field variations measured sufficiently close to the location where the GIC is computed, a simple and practical way to estimate it is to assume a downward-propagating plane wave impinging on either a uniform or a layered Earth. In this plane wave model, the electric and magnetic fields are horizontal and spatially constant at the Earth's surface. If, in addition, the Earth is uniform, a time-domain integral relation can be obtained, which easily allows for the use of magnetic observatory data to derive the GIC (e.g., Pirjola 2002; Ngwira et al. 2011; Torta et al. 2012). Note that in Ngwira et al. (2011) and Torta et al. (2012), the derivative of $B$ must be with respect to $u$ rather than with respect to $t$ :

$$
E_{x, y}(t)= \pm \frac{1}{\sqrt{\pi \mu_{0} \sigma}} \int_{-\infty}^{t} \frac{1}{\sqrt{t-u}} \frac{d B_{y, x}(u)}{d u} d u
$$

where $x$ and $y$ indicate the north and east geographical directions, respectively; $\mu_{0}$ is the permeability of free space; and $\sigma$ is the Earth's conductivity. The ' + ' sign on the right-hand side applies to $\left(E_{x}, B_{y}\right)$ and the '-' sign to $\left(E_{y}, B_{x}\right)$. In contrast, if one considers a layered Earth structure, the surface impedance $Z(\omega)$ can be obtained for each angular frequency $\omega$ by a recursive formula that determines the impedance at the top of any layer in terms of the impedance at the bottom of the layer (see, e.g., Pulkkinen 2003). The geoelectric field in the time domain is then evaluated by the inverse Fourier transform of

$$
E_{x, y}(\omega)= \pm \frac{Z(\omega) B_{y, x}(\omega)}{\mu_{0}},
$$

where $B(\omega)$ is the Fourier transform of the magnetic field (e.g., Viljanen et al. 2006). The integral in Equation 2 can be easily obtained numerically (Viljanen and Pirjola 1989). In evaluating that integral in practice, the lower limit of integration of Equation 2 is set to $t-M$, where $M$ is the amount of time taken into account before $t$ (for which the electric field is required). If this interval is sufficient, the time-dependent method (valid for a uniform Earth) provides the same results as the spectral method (valid for the layered Earth) provided that all the layers are given the same conductivity.

As to what concerns the engineering problem, there are two basic configurations of conductor networks: those corresponding to buried pipelines, which exhibit continuous contact with the ground and have to be solved, for example, by the use of the distributed source transmission line theory (e.g., Trichtchenko and Boteler 2002); and those corresponding to power transmission grids, which have discrete earthing points at the transformers. The latter can been solved using either the Mesh Impedance 
Matrix (MIM) method or the Nodal Admittance Matrix (NAM) method, which are used by the power industry (e.g., Albertson 1989), or the Lehtinen-Pirjola (LP) method (Lehtinen and Pirjola 1985), which has been used traditionally by the geophysics community. Actually, NAM and LP are the same, with the exception that NAM cannot deal with the interaction between the nodes described by the off-diagonal elements of the earthing impedance matrix in LP (see next paragraphs). The calculation technique in the MIM method is different but equivalent with NAM and LP in the sense that it is also based on circuit analysis and Ohm's and Kirchhoff's laws (R. Pirjola, personal communication). Following with that tradition, we used the LP method as well. Knowing that GICs are slowly varying compared to the $50-\mathrm{Hz}$ mains, a dc treatment is acceptable, and so we simply used Ohm's and Kirchhoff's laws in the dc form to formulate a network matrix model from the earthing/transmission line resistances provided by industry data. Lehtinen and Pirjola (1985) provided all the expressions that lead to the determination of the GIC (I) flowing into the Earth (or into the grid) at each of the $n$ nodes. They can be summarized in the following equation:

$$
\mathbf{I}=(\mathbf{1}+\mathbf{Y Z})^{-1} \mathbf{J},
$$

where $\mathbf{1}, \mathbf{Y}$, and $\mathbf{Z}$ are the identity matrix, the $n \times \mathbf{x} n$ network admittance (related with the line resistances) matrix, and the earthing impedance (related with the earthing resistances) matrix, respectively; the $n$-element vector J contains information on the electric field computed, as described before, from geomagnetic observatory data.

The elements of the $\mathbf{Y}$ and $\mathbf{Z}$ matrices are computed according to (Lehtinen and Pirjola 1985)

$$
Y_{i j}=\left\{\begin{array}{ll}
-\frac{1}{R_{i j}}, & \mathrm{i} \neq j \\
\sum_{k \neq i} \frac{1}{R_{i k}}, & i=j
\end{array},\right.
$$

where $R_{i j}$ is the line resistance between nodes $i$ and $j$, and if the nodes are far apart,

$$
Z_{i j}= \begin{cases}0, & i \neq j \\ R_{i}^{e}, & i=j\end{cases}
$$

where $R_{i}^{e}$ is the resistance through which the node $i$ is earthed. Pirjola (2008a, b) demonstrated that accounting for the off-diagonal elements of this matrix can be only relevant when the distances between the stations are on the order of hundreds of meters or less. In the Spanish $400-\mathrm{kV}$ network described in the next section, only three node links are separated by less than $500 \mathrm{~m}$ (between approximately 350 and $450 \mathrm{~m}$ ), so all the off-diagonal elements of the earthing impedance matrix were set equal to zero. In any case, neglecting all off-diagonal elements of $\mathbf{Z}$ is in general less important for the knowledge of the expected GICs than the uncertainties produced by the different approximations and assumptions adopted because it is not always possible to get precise values for all the circuit elements.

The $\mathbf{J}$ vector in Equation 4 is given by

$$
J_{i}=\sum_{j \neq i} \frac{V_{i j}}{R_{i j}}
$$

where $V_{i j}$ is the geovoltage from node $i$ to node $j$, which is given by

$$
V_{i j}=\int_{L_{i j}} \boldsymbol{E} \cdot d \boldsymbol{l},
$$

where $\boldsymbol{E}$ is the electric field, $\boldsymbol{d} \boldsymbol{l}$ is the differential vector path length, and the integration path $L_{i j}$ refers to the transmission line from $i$ to $j$. If $\boldsymbol{E}$ is conservative, the integral in Equation 8 is independent of the path $L_{i j}$ from $i$ to $j$, and $V_{i j}=V_{i}-V_{j}$, where $V_{i}$ and $V_{j}$ are the values of the scalar potential of the geoelectric field at nodes $i$ and $j$. A particular case of a conservative field is when it can be considered spatially constant in the region of analysis. Then, the GIC values in each node can be expressed in terms of two parameters ( $a$ and $b$ ), which depend on the geometry and the resistances of the network (and naturally vary from node to node):

$$
\operatorname{GIC}(t)=a E_{x}(t)+b E_{y}(t) .
$$

In summary, the external (or geophysical) conditions are contained in $\left(E_{x}, E_{y}\right)$, while the engineering problem is determined by the scalar constants $a$ and $b$. Unless one evaluates continental regions (Viljanen et al. 2012), the Earth's curvature can be neglected and planar geometry (rather than spherical) can be used to derive these constants. Horton et al. (2012) even consider the fact that the Earth is not exactly a sphere but an ellipsoid (utilized in global positioning system (GPS) applications that power companies also use).

\section{Results and discussion \\ Data and results}

In Torta et al. (2012), we presented the results obtained in a relatively small subset of the Spanish power transmission network, which corresponded to Catalonia and adjacent substations in the north-easternmost sector. We have now completed an assessment of the GIC vulnerability across the entire Spanish system. In the first approximation made to obtain the circuit model that will allow for the calculation of GICs, we analyzed the 400-kV network only. This includes 173 substations, 375 transformers, and 300 transmission lines (Figure 1). Only border foreign substations were included in the 


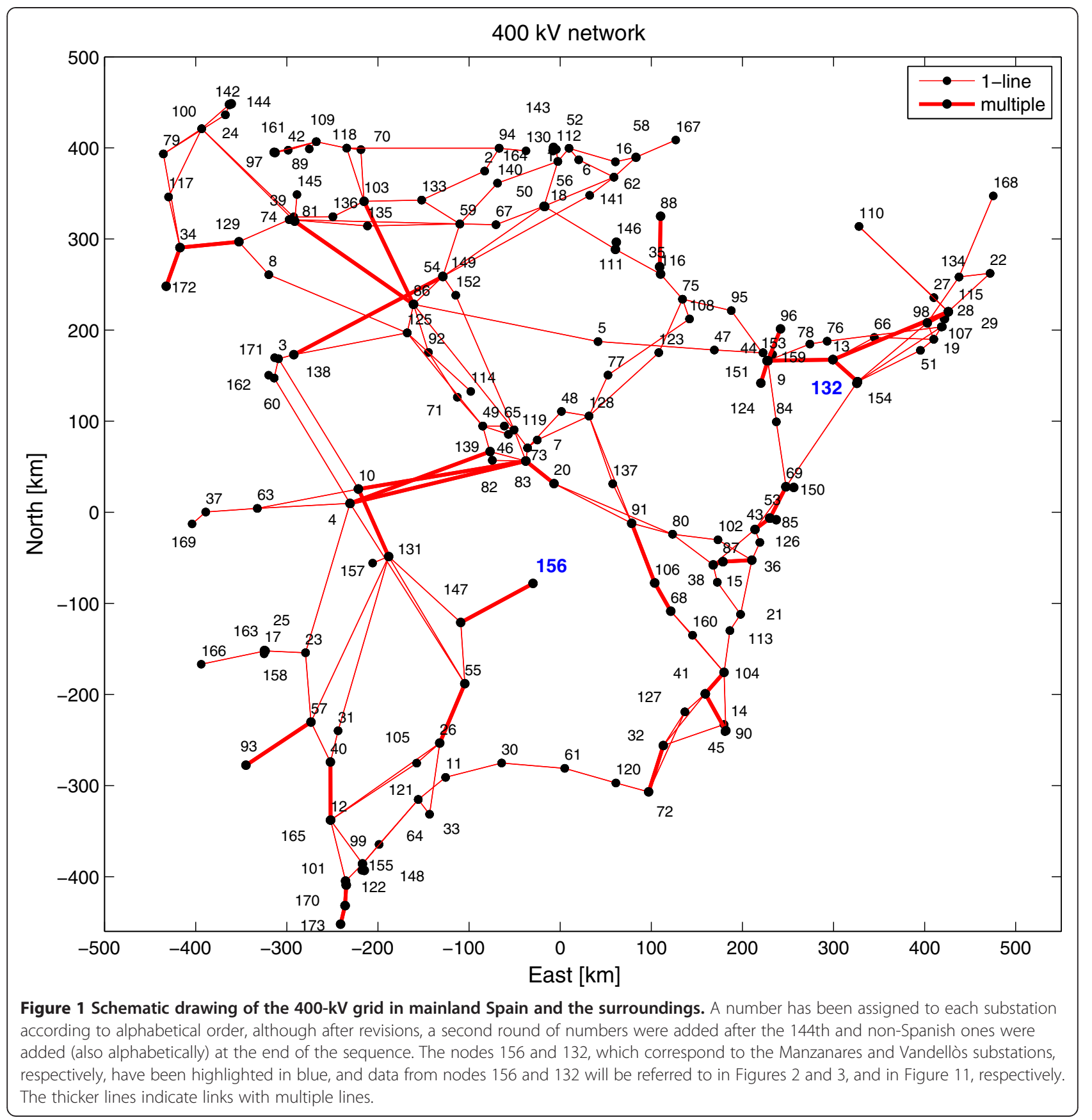

model, with the exception of Mellousa (the second in Morocco) because the first one (Fardioua) is a station without transformers. This is a common practice (e.g., Myllys et al. 2014) because it has been shown (Boteler et al. 2013) that the best choice for an equivalent circuit of the neighboring network comprises the induced voltage and resistance for the first transmission line into the neighboring system, so that it can be represented as the line to the first substation and its resistance to ground.

Information relating to the resistances of all the transformers is still incomplete, so that, for simplification, we completely ignored the autotransformers' low-voltage circuits, whereby the winding resistance is the sum of the common and the series resistances. For calculating the total earthing resistance at each substation, we considered the three phases in parallel and added the substation earthing resistance to their equivalent resistance. If the substation is composed of more than one transformer, we assumed that the transformers are connected in parallel and share the same substation earthing resistance. The values of these earthing resistances are in general unknown, but we assumed that they have a typical 
value of $0.15 \Omega$ (private communication with related power engineers), except for those few substations of the Catalan subset for which we obtained a different value from our previous project. Similarly, in the cases where the resistance of the windings of transformers is unknown, following the criterion of Viljanen et al. (2012), we assumed that $R_{i}^{e}$ takes a typical value of $0.5 \Omega$, which implies a total resistance for three parallel windings of $0.35 \Omega$, or $1.05 \Omega$ for one winding. The phase resistances of the power transmission links were calculated according to the values of the resistance per unit length of the conductors at $20^{\circ} \mathrm{C}$ and the number of conductors per phase supplied by the power company. Then, one must take into account the reduction of three phases into a single conductor.

As described in the previous section, the $\mathbf{Y}$ and $\mathbf{Z}$ matrices computed from the above resistance data allow for the GIC computation at each substation. Accordingly, we obtained a prediction (retrospective in this case) of the GICs in the network nodes and transformers for the Halloween storm and the 24 March 1991 storm, which displayed the most abrupt solar storm commencement at the Ebre Observatory (Torta et al. 2012). We assumed the current network configuration and all elements in operation. Figures 2 and 3 show the resulting GIC in the transformer that we identified as the most vulnerable because it was isolated at the end of a line. On occasion of the abovementioned events, in this transformer, we computed 70 and more than $100 \mathrm{~A}$, respectively. In fact, GICs have been calculated as functions of time through the entire Halloween storm of 29 to 31 October 2003, and the maximum absolute value for each individual substation has been taken and plotted in the map of Figure 4 as circles with diameter proportional to the absolute value of the current. This means that the maxima shown in Figure 4 were not necessarily obtained at the same time point. Large GICs were found in the stations located at the end, or in the corners of transmission lines as intuitively expected and shown by the explicit model calculations for the GIC (e.g., Viljanen and Pirjola 1994; Wik et al. 2008).

In order to analyze in a more generic way how the different directions of the electric field affect the GICs generated in the network, we applied a synthetic electric field of modulus equal to $1 \mathrm{~V} / \mathrm{km}$ in all directions. This value is slightly higher than the maximum value in the geomagnetic records of the Ebre Observatory (i.e., $0.73 \mathrm{~V} / \mathrm{km}$, which occurred during the storm of 24 March 1991), but it is a reasonable value for a particularly severe geomagnetic storm scenario and can always easily be scaled to correspond to any other uniform-field value (Pirjola 2008a). The obtained results are shown in Figure 5, and these represent those directions in which the maximum GIC flowing from the network to the Earth is reached at each node. The north corresponds to an angle of $0^{\circ}$ and the east to an angle of $90^{\circ}$. The length of the vectors indicates the maximum magnitude, and one must take into account that it is reached both in the given direction and in the opposite direction because a field reversal only changes the signs of the GICs (see Equation 9). In any case, as indicated by Bernabeu (2013), their impact on driving transformers into half-cycle saturation is independent of the direction of the current flow. Only those nodes that would achieve significant currents have been labeled (with the name of the substation and an indication of the number ascribed to them in Figure 1). From Figure 5, it can be deduced that the maximum GICs are achieved for those events in which the

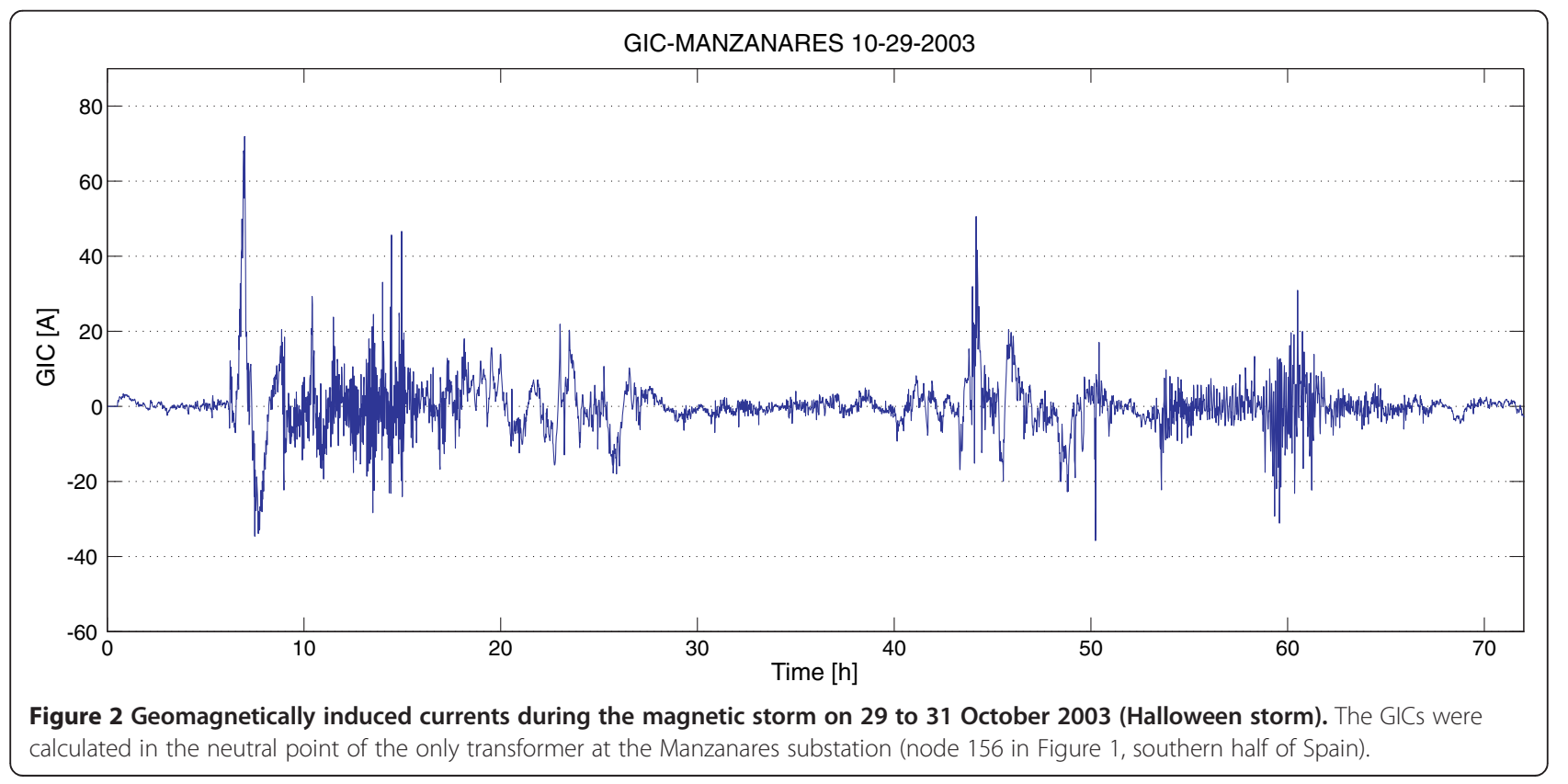




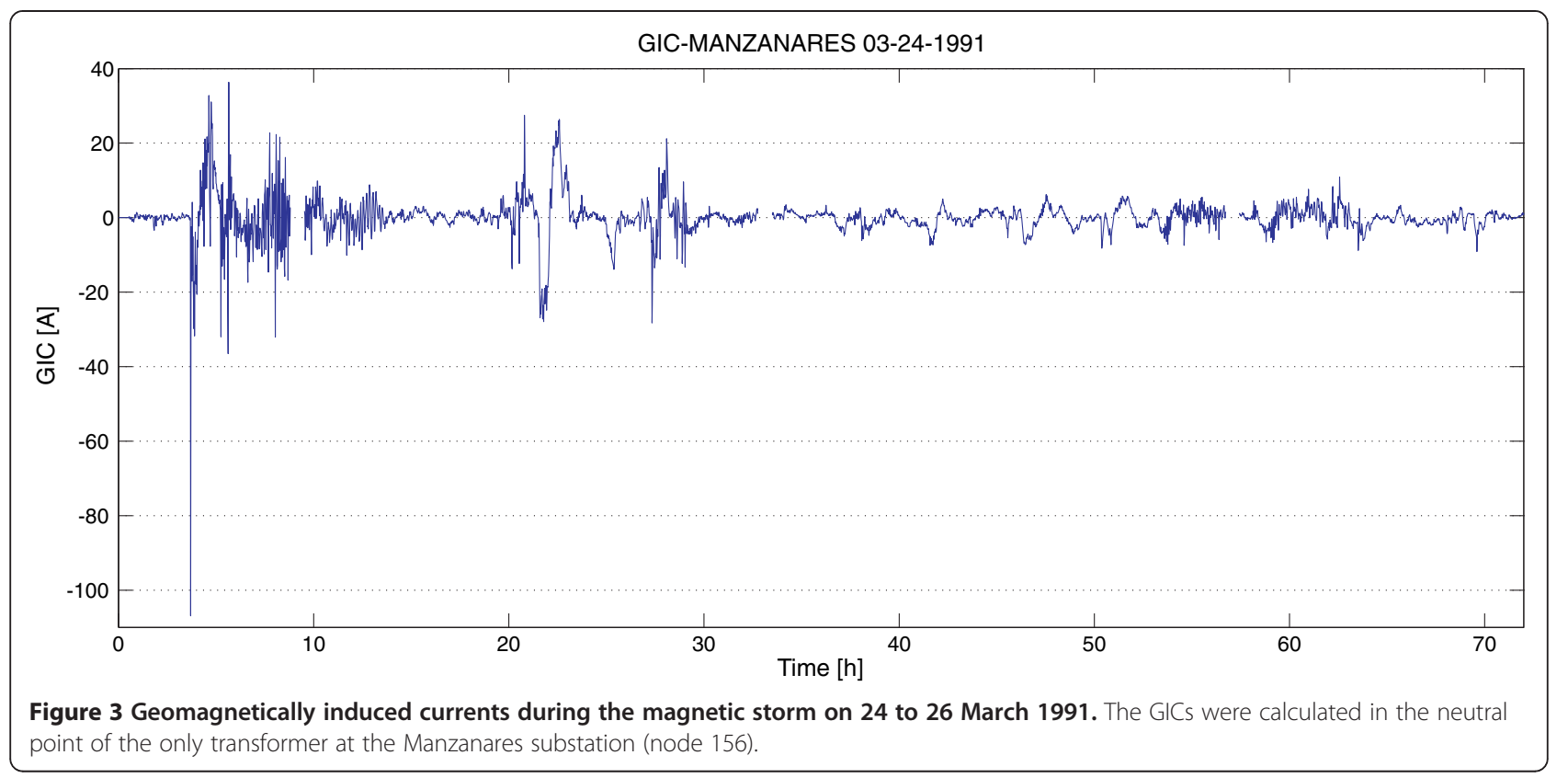

electric field direction coincides with the directions of the transmission lines. Given the geometry of the Spanish network, these directions are mostly NW-SE and ENE-WSW, and attention should be drawn to the magnitude of the GICs reached at Muruarte and Litoral substations, especially at the latter when the field is in the NNE-SSW direction. In the same way, Figure 6 was obtained by dividing the GIC values shown in Figure 5 among the transformers of each substation according to the corresponding current divider (i.e., in a ratio depending on the transformer resistances). Looking only at the total GIC value at a substation sometimes gives a good idea of the possible GIC hazard, which was the case, for example, at Manzanares (node 156 in Figure 1) and Brazatortas (node 147), which had just

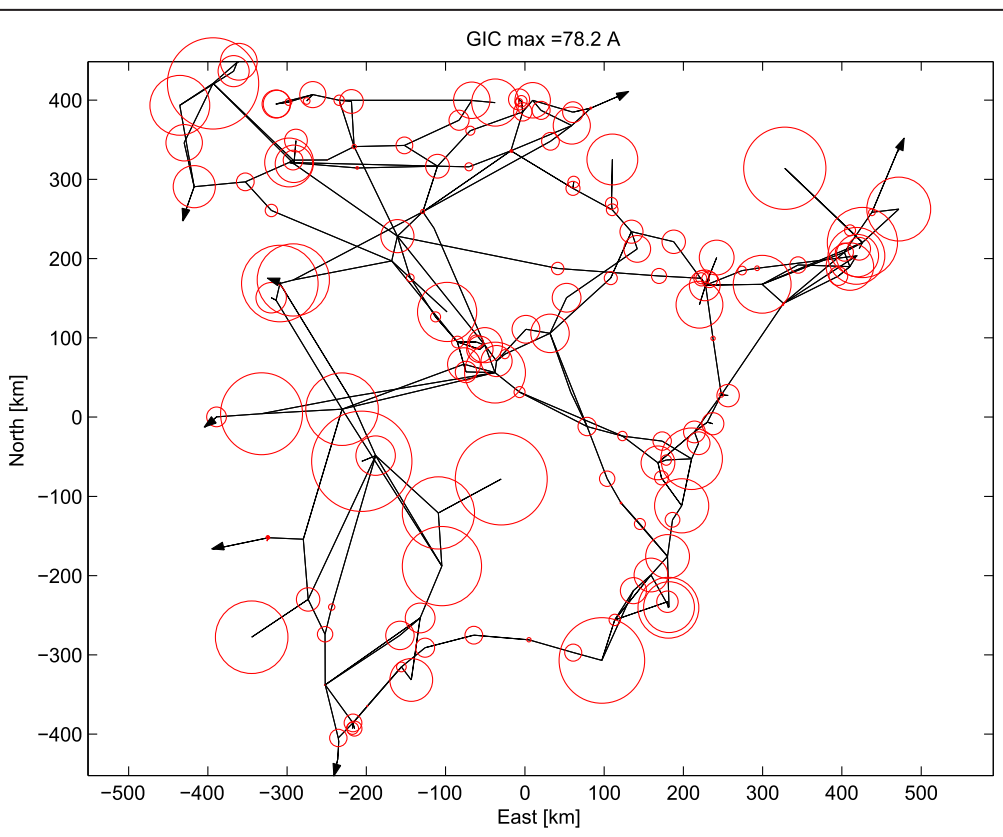

Figure 4 Maximum values of GICs under the conditions during the Halloween storm. Circles have diameters proportional to the flowing current (regardless of its sign), and a maximum of 78.2 A was observed, which was achieved at the Mesa de la Copa substation (node 157 in Figure 1). Arrowheads at the ends of some transmission lines indicate connection with foreign substations. 


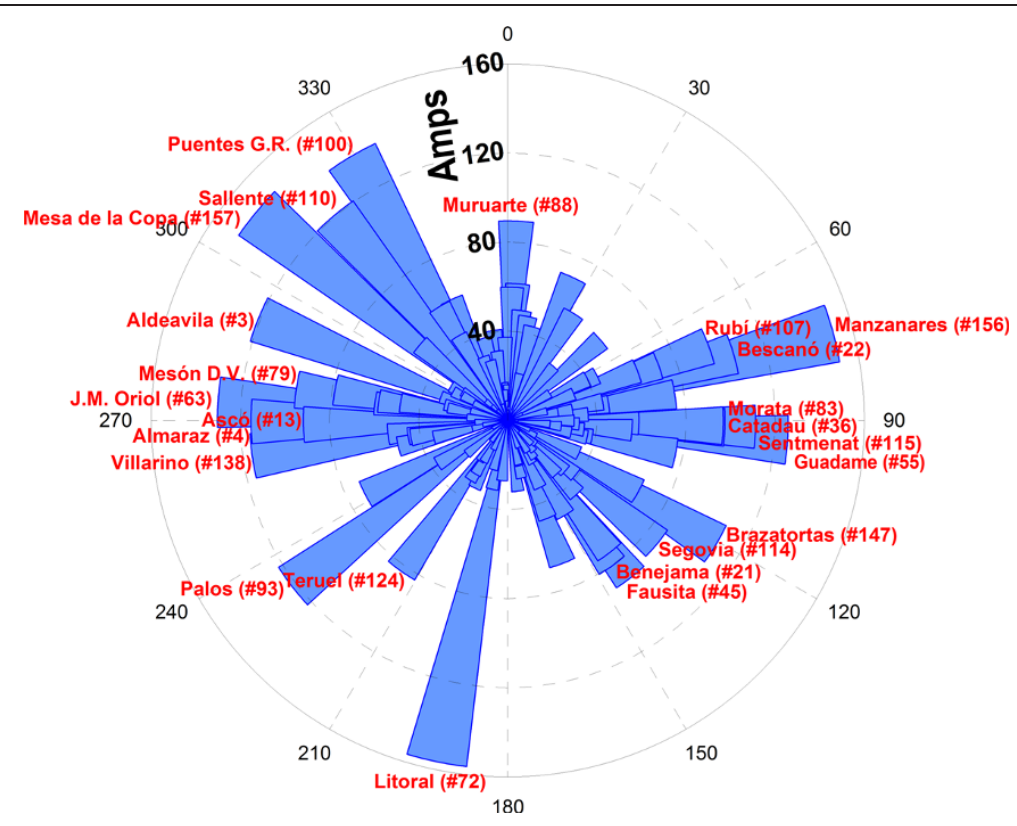

Figure 5 Directions in which the maximum GIC is obtained in each node. They correspond to GICs from the network to the Earth when a horizontal electric field of $1 \mathrm{~V} / \mathrm{km}$ is applied. The length of each bar indicates the maximum current.

one transformer. However, this practice sometimes overestimated the possible GIC hazard, which was the case at Litoral and Puentes G. R., which had several transformers.

Just as in the previous paragraph, in Figures 7 and 8, we show how GICs affect the Spanish network under the impact of a northward (Figure 7) and eastward (Figure 8) geoelectric field of $1 \mathrm{~V} / \mathrm{km}$. This value does not accurately represent the real situation, as the field is never homogeneous; it varies both due to spatial variations of the external geomagnetic field (especially, depending on the latitude) and due to spatial variations of the field created by currents induced in the crust and upper mantle (because of the remarkable heterogeneity in Earth's conductivity). But it is a reasonable magnitude for an extreme

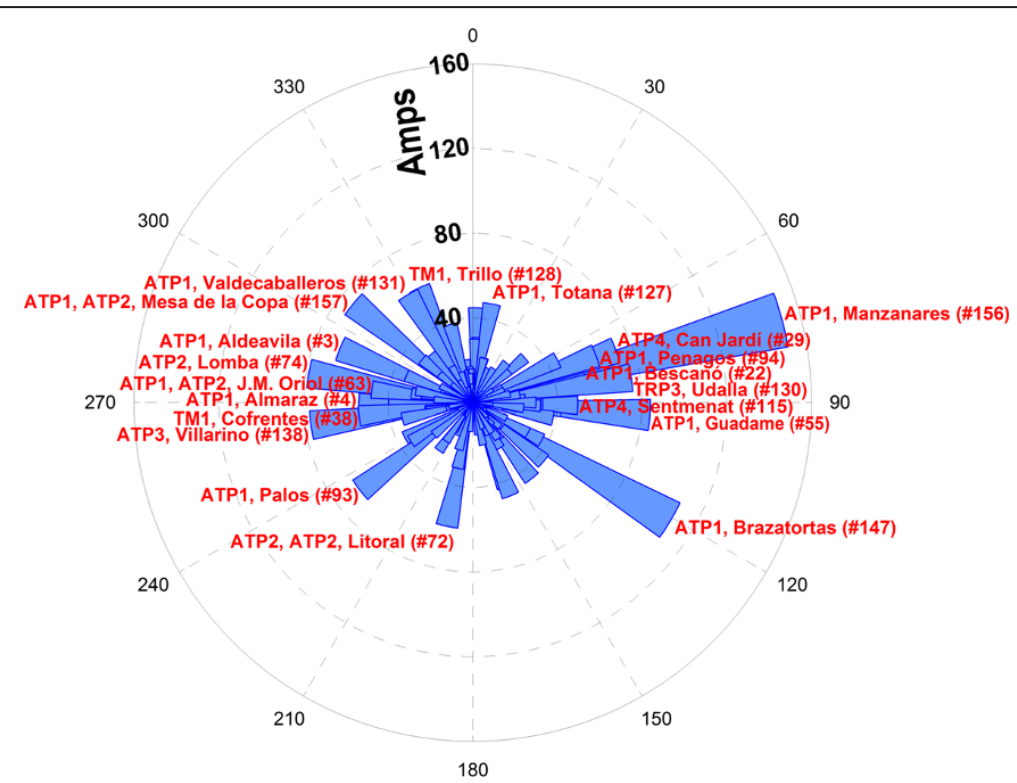

Figure 6 Directions in which the maximum GIC is obtained in each transformer. They correspond to GICs from the network to the Earth when a horizontal electric field of $1 \mathrm{~V} / \mathrm{km}$ is applied. The length of each bar indicates the maximum current. 


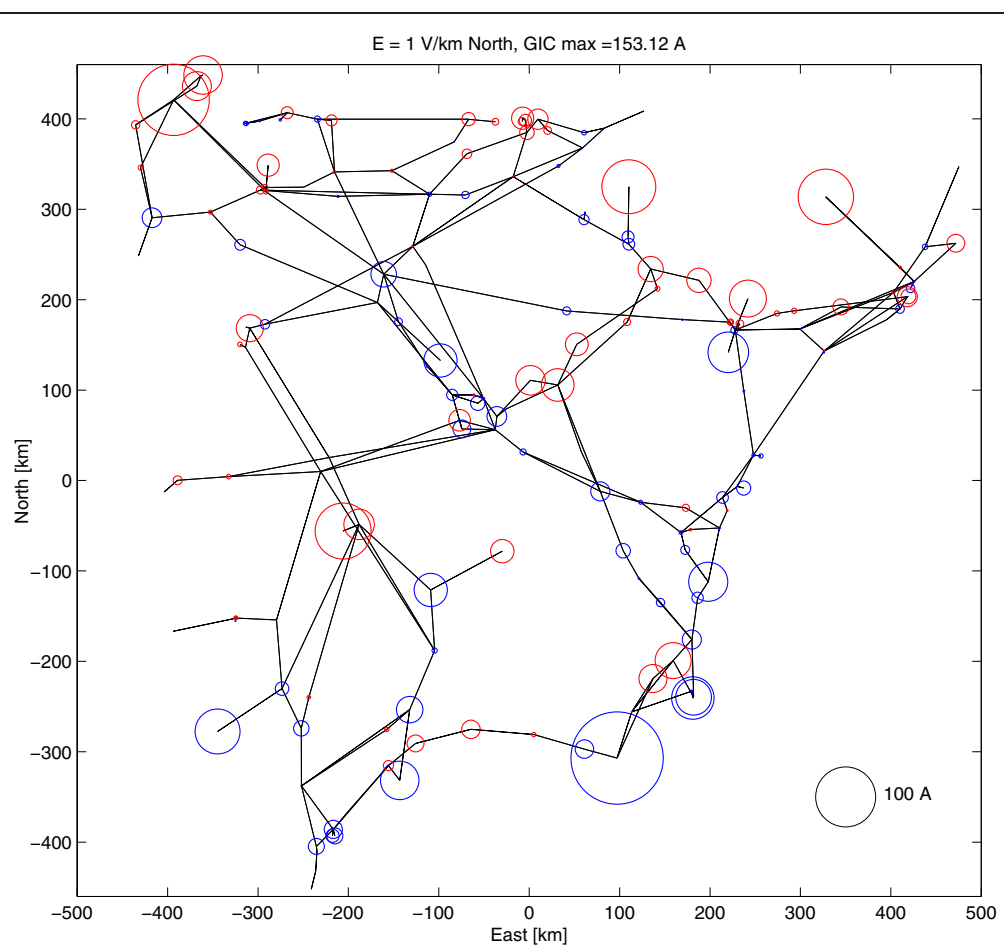

Figure 7 Maximum GICs due to a horizontal geoelectric field of $1 \mathrm{~V} / \mathbf{k m}$ in the northern direction. Data are shown in the form of circles, and the diameters are proportional to the flowing current. The red color denotes GICs flowing into Earth, and blue denotes those flowing into the grid.

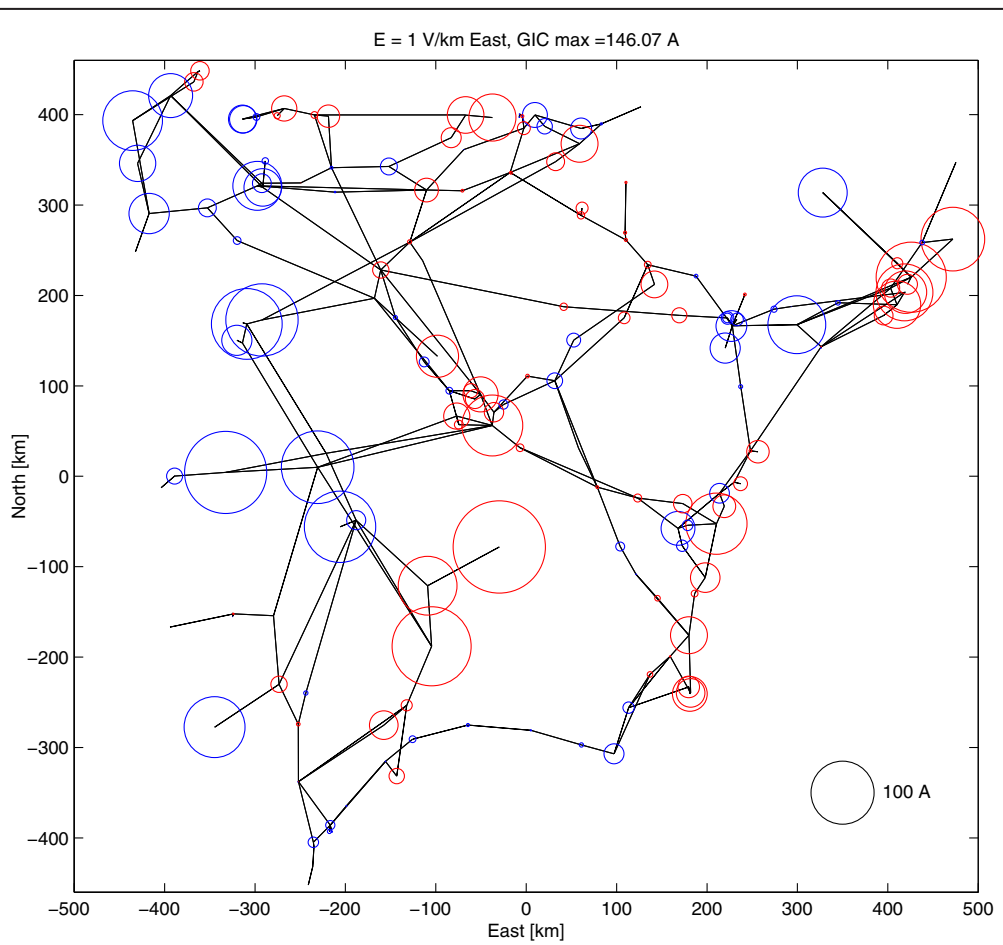

Figure 8 Maximum GICs due to a horizontal geoelectric field of $1 \mathrm{~V} / \mathrm{km}$ in the eastern direction. Data are shown in the form of circles, and the diameters are proportional to the flowing current. The red color denotes GICs flowing into Earth, and blue denotes those flowing into the grid. 
geomagnetic storm at the latitudes of interest of this study, and that selection is actually unimportant for the purpose of analyzing the vulnerability of the network because, as pointed out above, the values of the GICs are scaled proportionally to the electric field (Pirjola 2008a).

Clearly, in a real situation, the geomagnetic field varies in any direction and, consequently, so does the geoelectric field. Thus, the generated GICs will be variable but in proportion to the amplitudes shown in Figures 7 and 8. What is unchanged is the realization, as indicated by Boteler (1994) and Pirjola (2008b), that the GICs are maximized when the incident electric field is in the direction of the bisector of the angle formed by two transmission lines arriving at a substation and minimized in the perpendicular direction. Thus, the transformers at intermediate stations along a long line are not particularly vulnerable to GICs, and therefore, in principle, they are not interesting sites to undertake measurements.

The next step was the estimation of the return period of a hypothetical extreme event. We relied on the results of Thomson et al. (2011), who, using a number of decades of 1-min mean data, provided the maximum values observed in the derivative for both the horizontal intensity and the declination of the magnetic field and the values that could be observed every 100 or 200 years depending on the geomagnetic latitude of different European geomagnetic observatories; these data were presented along with the confidence limits at the 95\% level. For their analysis, they used a Generalized Pareto Distribution (GPD) to describe the tail of the distribution of geomagnetic activity. Before fitting each GPD to the data, they discarded data located below a certain threshold and isolated the magnetic field of external origin by subtracting the average level on a quiet day (set for each month of the year), thereby obtaining the residual intensity $B_{H}$ and declination $B_{D}$. Meanwhile, they grouped extreme events that were less than $12 \mathrm{~h}$ apart (taking the maximum value for the period) on the grounds that they were part of the same geomagnetic storm event. For each observatory, they computed the peak residual and peak rate-of-change predicted by the observatory GPD to be exceeded within the periods of 100 and 200 years, which was accomplished via the examination of the return-level statistics. The results of
Thomson et al. (2011) appear excessively high for the Ebre Observatory compared with those from observatories of similar geomagnetic latitude. This may have happened because the data used still contained artificial peaks in 2001. The predictions made by Thomson et al. (2011) were recalculated after we provided them with geomagnetic data that were reviewed for the period 2000 to 2010 (Table 1); this reanalysis yielded an extreme value of $308 \mathrm{nT} / \mathrm{min}$ for $d B_{H} / d t$ during the return period of 100 years. This value was used to obtain an approximate size of the GIC magnitudes that would flow in each of the transformers of the grid in the event that the extreme value actually occurred during the return period of 100 years at the Ebre Observatory, and we assumed an impulsive event at a cadence of $1 \mathrm{~min}$ along the geomagnetic North (Figure 9). As indicated by Torta et al. (2012), the northward impulse is the most plausible scenario for the most severe events at Ebre Observatory's latitude, where they tend to occur coincident with the abrupt onset of geomagnetic storms.

\section{Errors by the omission of low-voltage circuits}

Although it is a common practice to treat the highestvoltage system as the first approximation, as we did in this study, the correcting terms needed to match the actual flowing currents in the interconnecting transformers will not be attainable until we get the necessary data associated with the lower-voltage systems. To illustrate the amount of error that can be expected by the omission of low-voltage circuits of the network, we used data from a test case that was released to validate the programs and procedures used by the scientific and technical community to model the GICs (Horton et al. 2012). Such a benchmark network contains many features found in real networks, such as different voltage levels, two- and three-winding transformers, autotransformers, multiple transmission lines in the same bus, and GIC-blocking devices (see Figure 10 for simplified expressions of the different resistance structures of transformers). It represents a hypothetical 20 bus network with 8 substations, including 15 transmission lines and transformers of 500 and $345 \mathrm{kV}$. To model the GICs, Horton et al. (2012) provide three sets of necessary resistance data, i.e., data for the substations, transmission lines, and transformers.

Table 1 The measured maximum and estimated 100-year and 200-year return levels at the Ebre Observatory

\begin{tabular}{lcclccc}
\hline Variable & Measured maximum & 100-year return level & Confidence limit & 200-year return level & Confidence limit \\
\hline$B_{H}$ & 552 & 706 & 511 & 1,358 & 770 & 534 \\
$d B_{H} / d t$ & 112 & 308 & 231 & 384 & 428 & 1,509 \\
$B_{D}$ & 0.87 & 1.42 & 0.81 & 2.59 & 1.67 & 320 \\
$d B_{D} / d t$ & 0.15 & 0.25 & 0.18 & 0.33 & 0.31 & 0.85 \\
\hline
\end{tabular}

For the residual horizontal intensity $B_{H}$ (in $\mathrm{nT}$ ), the residual declination $B_{D}$ (in degrees), and their derivatives (in $\mathrm{nT} / \mathrm{min}$ and degrees/min, respectively); data are shown along with their $95 \%$ confidence limits. 


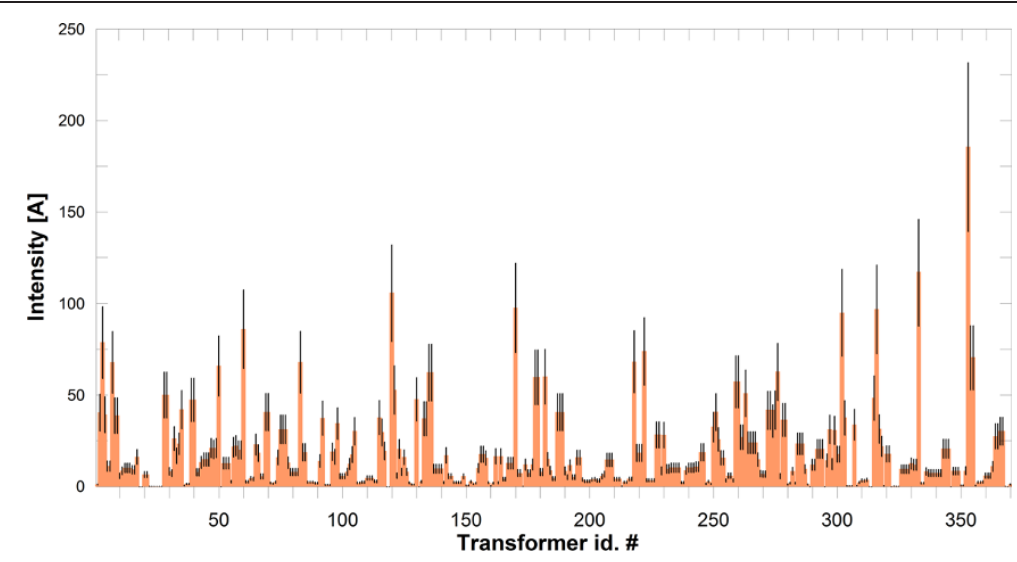

Figure 9 Maximum values of GICs in each transformer as a consequence of an extreme geomagnetic storm. They correspond to a scenario for a return period of 100 years. Here, we assume an impulsive event along the geomagnetic North. Vertical black lines indicate the $95 \%$ confidence levels.

They also provide geographical positions of the substations (from which one can calculate distances between them in kilometers).

Horton et al. (2012) provide the calculated GIC results in the network for two geoelectric field scenarios: again, a northward and an eastward uniform electric field of $1 \mathrm{~V} / \mathrm{km}$. It was found that we could obtain the same results with our programs. To achieve this, in the case of autotransformers, we had to make use of the concept of virtual nodes (Mäkinen 1993; Pirjola 2005) with an infinite resistance to ground and a resistance of a virtual line between the virtual and the real node equal to the series resistance of the actual autotransformer, while the common resistance is the resistance of the real transformer at the neutral point. Similarly, for full-wound transformers with a Y configuration and grounded both at high- and low-voltage sides, we used an equivalent circuit with two virtual nodes, one in each side of the transformer. Here, again, the virtual nodes have infinite resistances to ground and the virtual line resistances have been set between the virtual and real nodes equal to the real resistances of the transformer windings. Meanwhile, the actual transformer winding resistance was set to zero because it was located at the neutral point of the full-wound transformer.

We performed test calculations by omitting the entire low-voltage circuit (345 kV in that case). The results for the GICs flowing to ground at each substation are shown in the two central columns after the column of names in Table 2, whereas those flowing in the phases of each of the transformer windings are shown in the same columns of Table 3 . In both tables, the values obtained by Horton et al. (2012) when both the high and the low voltage are considered are also shown (first two columns), along with the percentage differences with respect to our test (last two columns). It is both clear and logical that the largest differences were obtained in substations that share lines of high and low voltage. The substations with autotransformers had the largest error (more than 200\% in some cases depending on the direction of the incident electric field), and at these locations, the GICs were usually overestimated when only the high-voltage circuit was modeled. The corrections of calculated GICs in the Spanish 400-kV network that are needed to account for the effects of neglecting the lower-voltage systems might be different. In any case, this test highlights the importance of having all the information concerning the positions and resistances of the network and the substation configurations, both for the high-voltage circuits and for the low-voltage ones, especially if there is galvanic connection between them.

\section{Comparisons with GIC measurements}

The best way to evaluate the performance of a model such as the one described here is to compare its predictions at a particular transformer neutral point with actual GIC measurements. This, although planned, is still not possible at key sites such as the transformer at the Manzanares substation. The only measurements coincident with relevant geomagnetic storms were those obtained in 2011 to 2012 on behalf of our previous project (Torta et al. 2012). We do compare here the real observations with the predictions of our new model, including the entire 400-kV Spanish network, for the same transformer named TRP1 (which was wrongly named as TR2 in Torta et al. 2012) at the Vandellòs substation (node 132 in Figure 1) on the occasion of the geomagnetic storm which occurred during 24 to 25 October 2011. Fortunately, there are no autotransformers in this substation, so it is not galvanically interconnected with low-voltage systems and thus we do not expect major errors by ignoring the $220-$ or $110-\mathrm{kV}$ networks at 


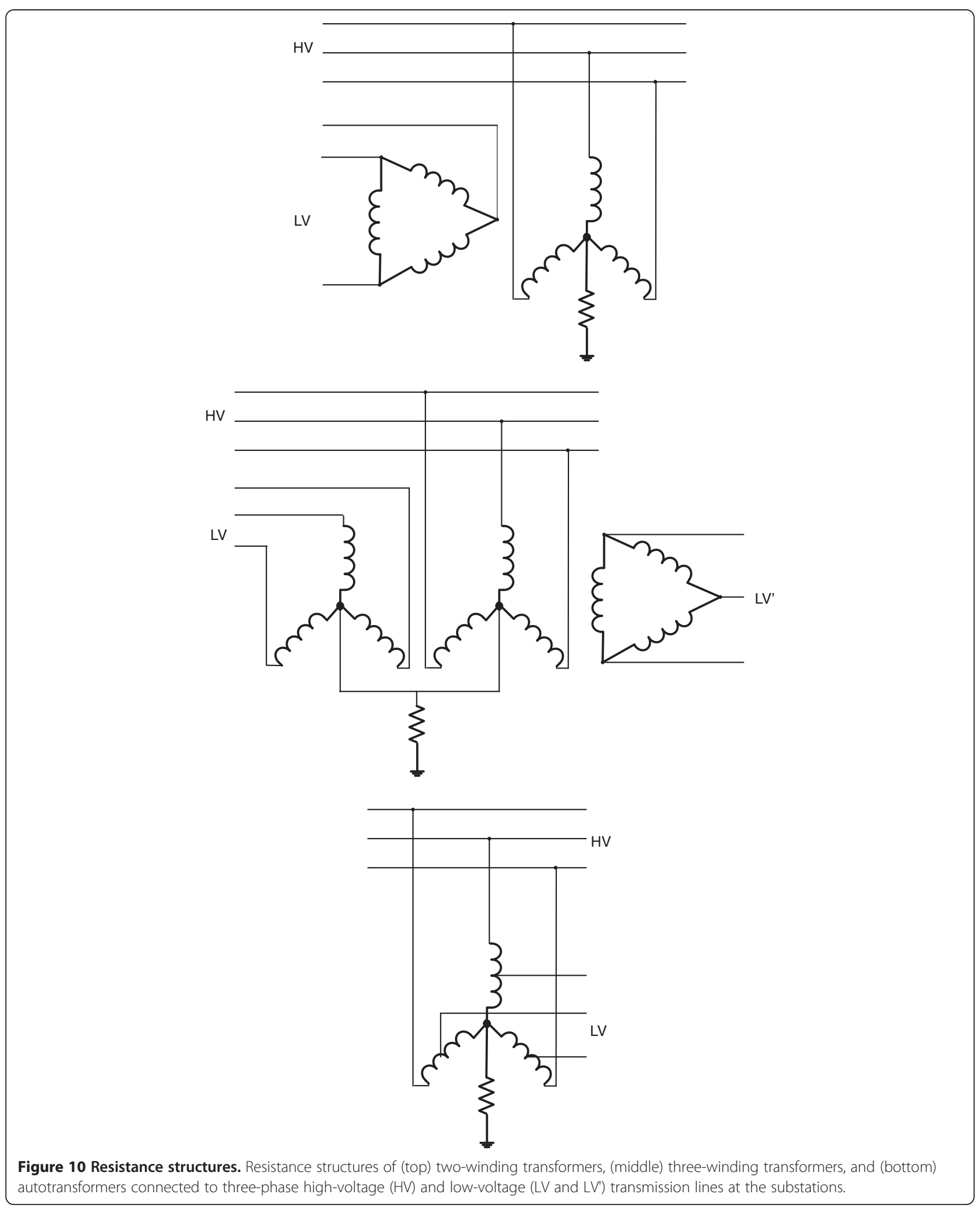


Table 2 Comparison of the total GICs flowing in the ground grid of each substation

\begin{tabular}{|c|c|c|c|c|c|c|}
\hline Name & $\begin{array}{l}\text { GIC for northward E } \\
\text { field (A) }\end{array}$ & $\begin{array}{l}\text { GIC for eastward E } \\
\text { field (A) }\end{array}$ & $\begin{array}{l}\text { GIC for northward } E \\
\text { field, } \mathrm{HV} \text { only }(\mathrm{A})\end{array}$ & $\begin{array}{l}\text { GIC for eastward E } \\
\text { field, HV only (A) }\end{array}$ & $\begin{array}{l}\text { GIC for northward E } \\
\text { field (\% diff.) }\end{array}$ & $\begin{array}{l}\text { GIC for eastward E } \\
\text { field (\% diff.) }\end{array}$ \\
\hline Sub 3 & 139.85 & -109.49 & 131.33 & -185.03 & 6.1 & 69.0 \\
\hline Sub 4 & 19.98 & -124.58 & 29.66 & -139.94 & 48.4 & 12.3 \\
\hline Sub 5 & -279.08 & -65.46 & -172.81 & -126.07 & 38.1 & 92.6 \\
\hline Sub 6 & -57.29 & 354.52 & -49.92 & 319.51 & 12.9 & 9.9 \\
\hline Sub 7 & 0.00 & 0.00 & 0.00 & 0.00 & 0.0 & 0.0 \\
\hline Sub 8 & 60.90 & 134.30 & 61.74 & 131.53 & 1.4 & 2.1 \\
\hline
\end{tabular}

The table shows the comparison of the total GICs flowing in the ground grid of each substation for the benchmark test case (Table VII of Horton et al. 2012) when a northward or eastward uniform electric field of $1 \mathrm{~V} / \mathrm{km}$ was present (first two columns after the column of names), and the GICs obtained using only the high-voltage circuit. The percent differences (\% diff.) data are also shown. HV stands for high voltage.

farther 400-kV stations (Pirjola and Viljanen 1991; Viljanen and Pirjola 1994; Pirjola 2005, 2010).

In Figure 11, we show the performance of our model as a function of the Earth conductivity structure that we used. We practiced either with a uniform ground conductivity of $\sigma=0.001 \mathrm{~S} / \mathrm{m}$ (as in Torta et al. 2012) or with different layered conductivity models. For the latter, we chose either the electrical resistivity model given by Pous et al. (1995) for the Ebre basin or the 1-D ground model of the \#29 block in Figure 2 by Viljanen et al. (2012). In Table 4, we give the linear correlation coefficients for each case, but in our opinion, this measure is not always the best indicator for quantifying the goodness of such model performances. This is because models that provide the same signal but are amplified several times will provide the same correlation coefficients. Thus, to evaluate how well the model fits the
GIC observations, we define the performance $(P)$ parameter as

$$
P=1-\frac{\mathrm{RMSD}_{o m}}{\sigma_{o}},
$$

where $\sigma_{o}$ is the standard deviation of the set of observations and $\mathrm{RMSD}_{\text {om }}$ is the root mean square deviation of the residuals (or differences between model predictions and observations). Here, the

$$
\operatorname{RMSD}_{o m}=\sqrt{\frac{\sum_{i=1}^{N}\left(o_{i}-m_{i}\right)^{2}}{N}}
$$

where $o_{i}$ and $m_{i}$ are the $i$ th observation and model output, respectively (or equivalently, those corresponding to time $t_{i}$ ), from a total of $N$. Given that the quotient in Equation 10 is a positively defined quantity, $P$ cannot be

\begin{tabular}{|c|c|c|c|c|c|c|c|}
\hline Name & Winding & $\begin{array}{l}\text { GIC for northward } \\
\text { E field (A/phase) }\end{array}$ & $\begin{array}{l}\text { GIC for eastward } \\
\text { E field (A/phase) }\end{array}$ & $\begin{array}{l}\text { GIC for northward } \\
\text { E field, HV only } \\
\text { (A/phase) }\end{array}$ & $\begin{array}{l}\text { GIC for eastward } \\
\text { E field, HV only } \\
\text { (A/phase) }\end{array}$ & $\begin{array}{l}\text { GIC for northward } \\
\text { E field (\% diff.) }\end{array}$ & $\begin{array}{l}\text { GIC for eastward } \\
\text { E field (\% diff.) }\end{array}$ \\
\hline $\mathrm{T} 2$ & $\mathrm{HV}$ & 1.75 & -6.94 & 1.65 & -7.77 & 5.8 & 12.0 \\
\hline T5 & Common & 23.31 & -18.25 & 21.89 & -30.84 & 6.1 & 69.0 \\
\hline T6 & $\mathrm{HV}$ & -9.55 & 59.09 & -8.32 & 53.25 & 12.9 & 9.9 \\
\hline T7 & HV & -9.55 & 59.09 & -8.32 & 53.25 & 12.9 & 9.9 \\
\hline T8 & $\mathrm{HV}$ & -27.67 & -17.89 & -28.80 & -21.01 & 4.1 & 17.5 \\
\hline T9 & HV & -27.67 & -17.89 & -28.80 & -21.01 & 4.1 & 17.5 \\
\hline $\mathrm{T} 10$ & $\mathrm{HV}$ & 10.15 & 22.38 & 10.29 & 21.92 & 1.4 & 2.0 \\
\hline T11 & HV & 10.15 & 22.38 & 10.29 & 21.92 & 1.4 & 2.0 \\
\hline T12 & Common & 0.99 & -8.64 & 3.30 & -15.55 & 232.9 & 80.0 \\
\hline T13 & HV & 1.75 & -6.94 & 1.65 & -7.77 & 5.8 & 12.0 \\
\hline T14 & Common & 0.99 & -8.64 & 3.30 & -15.55 & 232.9 & 80.0 \\
\hline T15 & Common & 23.31 & -18.25 & 21.89 & -30.84 & 6.1 & 69.0 \\
\hline
\end{tabular}

Table 3 Comparison of the total GICs flowing in the transformer windings

The table shows the comparison of the total GICs flowing in the transformer windings for the benchmark test case (Table VIII of Horton et al. 2012) when a northward or eastward uniform electric field of $1 \mathrm{~V} / \mathrm{km}$ was present (first two columns after the column of names). The percent differences (\% diff.) data are also shown. HV stands for high voltage. 


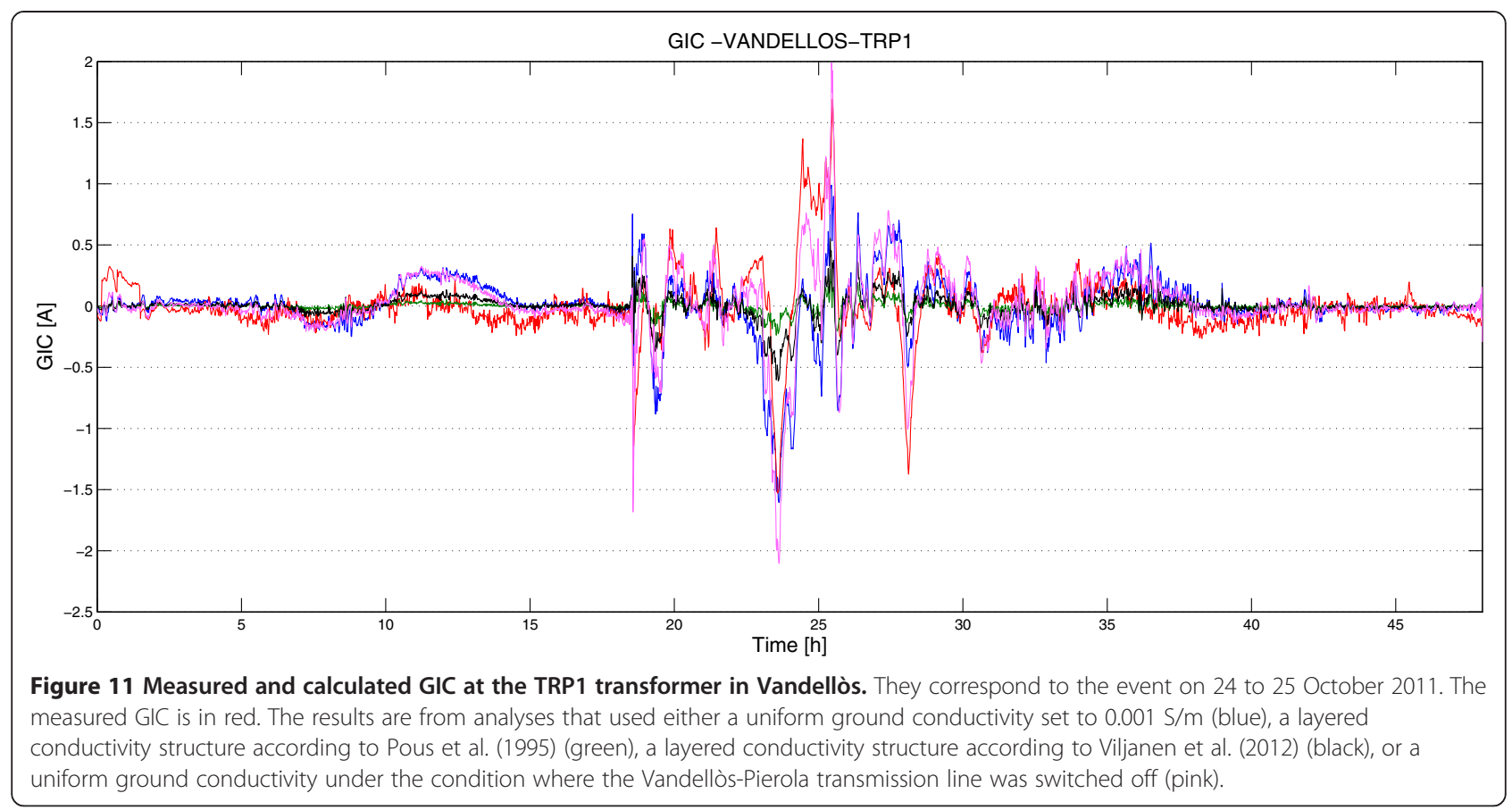

greater than 1 , which is reached when the residuals are zero, i.e., when the model exactly fits the observations. Otherwise, $P<1$. $P$ would be 0 , for example, for a flat model output equal to the mean of the observations. A negative value of $P$ usually (though not necessarily) denotes anti-correlation. So, in summary, models that perform better have higher values of $P$, and one can think of $P$ (with some reservations) as the fraction of the standard deviation of the observations that can be explained by the model. Results for this parameter are also given in Table 4 for each of the conductivity case structures tested. None of the proposed 1-D conductivity structures performed substantially better than the homogenous Earth approach, which suggests, as expected, that the actual structure must be laterally heterogeneous, especially because the lateral conductivity contrast is large at ocean-land interfaces (Beggan et al. 2013; Püthe and Kuvshinov 2013); this

\section{Table 4 Quantification of the goodness of different model predictions}

\begin{tabular}{lcc}
\hline Earth conductivity structure & $\boldsymbol{\rho}$ & $\boldsymbol{P}$ \\
\hline Uniform & 0.372 & 0.051 \\
Layered (Pous et al. 1995) & 0.368 & 0.045 \\
Layered (Viljanen et al. 2012) & 0.377 & 0.074 \\
Uniform (under the condition where the Vandellòs-Pierola & 0.749 & 0.252 \\
line was switched off) & &
\end{tabular}

The table shows the quantification of the goodness of the different model predictions with respect to the GIC measurements at the TRP1 transformer in the Vandellòs substation for the 24 to 25 October 2011 event according to the linear correlation coefficient, $\rho$, and the performance parameter $P$. makes the 1-D assumption probably invalid in the coastal area.

Since the calculated GIC at one site is slightly sensitive to changes made at distant points of the network (Pirjola 2009), our results can be reasonably compared with those presented in Figure 6 by Torta et al. (2012), when only a subset of the grid was modeled. It is well known that the load and operating procedure of the grid change over time. When we prepared our previous model, we did not have the exact elements of maintenance conditions (switched off) for the occasion of the geomagnetic storm, so we had to resort to the state of the grid that happened on a particular day about 3 months later, in which the lines Ascó (node 13 in Figure 1)-Pierola (node 98), Vandellòs (node 132)-Pierola (node 98), and Vandellòs (node 132)-Rubí (node 107) were switched off. Now, Red Eléctrica de España has provided us with updates regarding the elements of maintenance during 24 to 25 October 2011. The first important realization was that, on that occasion, one of the transformers at Vandellòs was switched off so that the total current produced by the geomagnetic storm could not be shared among the two transformers at that substation; hence, it only flew through the neutral point of the TRP1 transformer. This explains why the predictions of Torta et al. (2012) were underestimated and they had to artificially decrease the ground conductivity to match the amplitudes of measured and modeled GICs. However, although the overall amplitudes of the present study agree with more realistic conductivity values, our present results correlate worse with the measures of Torta et al. (2012). There are two explanations for this. The first 
one lies in the fact that Torta et al. (2012) used the timedomain integral approach to infer the electric field from the geomagnetic variation data by setting $M$ equal to just $30 \mathrm{~min}$. This converted the data into a surprising result because one would expect that the spectral method or the time-domain method with a sufficiently high $M$ should give a better fit for the GIC data (in this study, we used values of $M$ around $300 \mathrm{~min}$, which yielded results similar to the spectral method). Using $M=30$ means that Torta et al. (2012) truncated the impulse response to include only fast variations (i.e., high frequencies). This, in combination with the Earth model that is perhaps not exactly correct, happened to lead to a good fit with measured GIC data (R. Pirjola, D. Boteler, personal communication). A second explanation is related to the topology of the grid in the vicinity of the measuring substation. Besides the second transformer at Vandellòs, the switched off elements in the present model just included the Vandellòs-Rubí transmission line (other switched off elements far apart are not prone to influence the currents at Vandellòs). If we omit again the Vandellòs-Pierola line, the correlation significantly improves (Table 4, last row). We suspect that, although not reported, this transmission line or the Vandellòs-Garraf (node 51 of Figure 1) line, which runs almost parallel, was not operating for at least some time during the storm.

\section{Conclusions}

There is no doubt that space weather effects are an emerging natural hazard, which can have incredibly important effects on our lives, which are marked by increasing levels of technological dependence. They are in the category of high-impact, low-frequency event risks. When space weather effects refer to induced currents in technological systems such as power transmission grids, they are called geomagnetically induced currents, or simply, GICs. Modeling efforts require a determination of the electric field occurring in connection with a magnetic storm at the Earth's surface. There are several possible ways to accomplish this task. Once the geoelectric field is obtained, one can calculate the resulting GIC in the conductor system after obtaining a dc model of it. This engineering task requires knowledge of the geometrical configuration of the network stations and their connections, and also the resistance values of the whole system.

This paper provides much useful information about the expected GIC values in the entire Spanish $400-\mathrm{kV}$ power transmission network, which enables us to conduct an assessment of the vulnerability from that hazard. The network contains 165 substations, to which we added 8 substations from France, Portugal, and Morocco across the respective borders. This represents a complex circuit with 375 transformers and 300 transmission lines.
After obtaining the electrical network model to evaluate the maximum expected GIC in each transformer as a result of extreme geomagnetic storms, we developed a post-event analysis from the geomagnetic data obtained at the Ebre Observatory during certain storms, such as the Halloween storm in 2003. We also analyzed other episodes coincident with very abrupt sudden storm commencements, as they have proven to be even more dangerous in mid-latitudes. We found, as expected, that the most susceptible substations to GICs are those in corners or edges of the network. A typical case is that of the Manzanares station which, by having a single transformer, makes it particularly vulnerable. Had the storm of 24 March 1991 occurred with the current network configuration and with all the elements operative, GICs would exceed $100 \mathrm{~A}$ in the neutral point of the transformer. However, this extreme value will surely be significantly reduced when transmission lines that are under construction join Manzanares with another station to the east and become operational.

Assessing the vulnerability of a system to GIC hazards usually does not require knowledge of the precise values of expected GICs, instead rough magnitude estimates are sufficient (Pirjola 2008a). However, substantial improvements in our enterprise could be attained with further insights. A clear source of uncertainty in the obtained results arises from the fact of having completely ignored the low-voltage circuits that are galvanically connected to the $400-\mathrm{kV}$ system through autotransformers. This was done because we had neither information on the $220 \mathrm{kV}$ network nor the separate value of the common and series winding resistances of these autotransformers. The 110$\mathrm{kV}$ grid obviously does not have a very big effect on the GIC in the 400-kV system since the lower the voltage, the higher the transmission line resistances, which makes the GICs smaller. The importance of the omission of the low-voltage network has been demonstrated through a test under a GIC benchmark network that has been recently developed by Horton et al. (2012).

To test the model performance on a substation close to the location of Ebre Observatory, where the geomagnetic data came from, a homogenous Earth and a couple of 1-D Earth conductivity models have been employed based on the sparse results of magnetotelluric surveys published in the geophysical literature. This could give rise to calculated geoelectric field amplitudes that may be scaled to incorrect values due to the lack of precise ground conductivity data. In the few sites in which GICs have been or will be effectively measured, the two geoelectric components should be given the correct relative weight when the calculated values are fitted to the measured GIC data (Pirjola 2009; Torta et al. 2012), but because the source field is not exactly a plane wave and the real Earth is not homogeneous, we must consider 
the specific value of proportionality between the GIC and the electric field only as an 'effective conductivity' (Viljanen 1998). Therefore, another improvement would be expected after realistically modeling the induced electric field on the surface of the Earth by using a 3-D model of Earth's conductivity, and this can be done in the manner of recent works such as those by Beggan et al. (2013), Dong et al. (2013), and Püthe and Kuvshinov (2013). In particular, Spain is located on a peninsula, so a precise estimation of the geoelectric field would benefit from modeling the effects of non-uniform conductivities produced by the coast (Gilbert 2005; Thomson et al. 2005; Bürstinghaus et al. 2013; Pirjola 2013).

A critical fact, nevertheless, is the difficulty of obtaining detailed network parameters in the precise instant of a geomagnetic storm and determining the topology of the network for a given amplitude of the incident field. The switching off of a key transmission line or transformer can be essential to boost significant currents through the remaining elements.

\section{Competing interests}

The authors declare that they have no competing interests.

\section{Authors' contributions}

JMT carried out the analyses of the different modeling approaches and the pretreatment of all the geomagnetic and power grid data, obtained both the model results and the comparison with GIC measurements, and drafted the manuscript. SM designed the performance parameter to evaluate the model performances and participated in the comparison with GIC measurements. He also contributed to the design of the software codes. MQ provided all the information about the positions of each substation and links, the resistances of the lines and substations, and the different transformer configurations at each substation. She also attended to all questions concerning the engineering aspects of the paper. All authors read and approved the final manuscript.

\section{Acknowledgements}

This research is part of the project 'Geomagnetically induced currents in the power transmission network,' which is funded by Red Eléctrica de España, S. A.U. The GIC measurement data at the Vandellòs substation were obtained by a previous project funded by ENDESA Distribución Eléctrica, S.L. We wish to thank Lluís Serrano, formerly at Ebre Observatory, for preparing most of the software used in this paper, and Ewan Dawson of the Geomagnetism Team, British Geological Survey, Edinburgh, for computing the values provided in Table 1. Some discussions with Risto Pirjola, David Boteler, and Ari Viljanen and their suggestions helped to improve our work. Finally, we wish to acknowledge Risto Pirjola and an anonymous reviewer for their valuable comments and constructive criticisms that greatly enhanced the quality of the manuscript.

\section{Author details}

'Observatori de l'Ebre, (OE), CSIC, Universitat Ramon Llull, Horta Alta 38, Roquetes 43520, Spain. ${ }^{2}$ Red Eléctrica de España, P. del Conde de los Gaitanes, 177, Alcobendas, Madrid 28109, Spain.

Received: 28 January 2014 Accepted: 16 July 2014

Published: 4 August 2014

\section{References}

Albertson VD (1989) Modeling and analysis of GIC. In: Proceedings of EPRI conference on geomagnetically induced currents, Burlingame, California, USA, 8-10 November 1989

Amm O (1997) lonospheric elementary current systems in spherical coordinates and their application. J Geomag Geoelectr 49:947-955
Beggan CD, Beamish D, Richards A, Kelly GS, Thomson AWP (2013) Prediction of extreme geomagnetically induced currents in the UK high-voltage network. Space Weather 11:407-419, doi:10.1002/swe.20065

Bernabeu EE (2013) Modeling geomagnetically induced currents in Dominion Virginia Power using extreme 100-year geoelectric field scenarios-part 1. IEEE Trans Power Deliv 28:516-523

Blanch E, Marsal S, Segarra A, Torta JM, Altadill D, Curto JJ (2013) Space weather effects on Earth's environment associated to the 24-25 October 2011 geomagnetic storm. Space Weather 11:153-168, doi:10.1002/swe.20035

Bolduc L (2002) GIC observations and studies in the Hydro-Québec power system. J Atmos Sol-Terr Phy 64:1793-1802

Boteler DH (1994) Geomagnetically induced currents: present knowledge and future research. IEEE T Power Deliver 9:50-58

Boteler DH, Pirjola RJ (1998) The complex-image method for calculating the magnetic and electric fields at the surface of the Earth by the auroral electrojet. Geophys J Int 132:31-40

Boteler DH, Lackey AJC, Marti L, Shelemy S (2013) Equivalent circuits for modelling geomagnetically induced currents from a neighbouring network. In: Proceedings of the 2013 IEEE power and energy society general meeting (PES), Vancouver, BC, 21-25 July 2013., doi:10.1109/PESMG.2013.6672982

Bürstinghaus EJ, Saha TK, Marshall RA, Yumoto K, Waters CL (2013) The importance of non-uniform geoelectric fields in calculating GIC distributions. In: Proceedings of IEEE power and energy society general meeting 2013, 21-25 July 2013., doi:10.1109/PESMG.2013.6672289

Caraballo R, Sánchez Bettucci L, Tancredi G (2013) Geomagnetically induced currents in the Uruguayan high-voltage power grid. Geophys I Int 195:844-853

Cliver EW, Dietrich WF (2013) The 1859 space weather event revisited: limits of extreme activity. J Space Weather Space Clim 3:A31, doi:10.1051/swsc/2013053

Dong B, Danskin DW, Pirjola RJ, Boteler DH, Wang ZZ (2013) Evaluating the applicability of the finite element method for modelling of geoelectric fields. Ann Geophys 31:1689-1698

Gaunt CT, Coetzee G (2007) Transformer failure in regions incorrectly considered to have low GIC-risk. Paper presented at Power Tech 2007. Inst of Electr and Electron Eng, Lausanne, Switzerland

Gilbert JL (2005) Modeling the effect of the ocean-land interface on induced electric fields during geomagnetic storms, Space Weather 3:S04A03., doi:10.1029/2004SW000120

Horton R, Boteler D, Overbye TJ, Pirjola R, Dugan RC (2012) A test case for the calculation of geomagnetically induced currents. IEEE Trans Power Deliv 27:2368-2373, doi:10.1109/TPWRD.2012.2206407

Kappenman JG (2006) Great geomagnetic storms and extreme impulsive geomagnetic field disturbance events - an analysis of observational evidence including the great storm of May 1921. Adv Space Res 38:188-199

Kappenman JG (2007) Geomagnetic disturbances and impacts upon power system operation. In: Grigsby LL (ed) The Electric Power Engineering Handbook, 2nd edn. CRC Press/EEE Press, Auburn, Alabama

Koen J, Gaunt CT (2002) Geomagnetically induced currents at mid-latitudes. In: Proc URSI 27th Gral Ass, Maastrich, Netherlands, 17-24 August 2002., p 177

Lehtinen M, Pirjola R (1985) Currents produced in earthed conductor networks by geomagnetically-induced electric fields. Ann Geophys 3:479-484

Mäkinen M (1993) Geomagnetically induced currents in the Finnish power transmission system. Geophysical publication n ${ }^{\circ} 32$, Finnish Meteorological Institute, Helsinki, Finland

Molinski TS (2002) Why utilities respect geomagnetically induced currents J Atmos Sol Terr Phys 64:1765-1778

Myllys M, Viljanen A, Rui ØA, Ohnstad TM (2014) Geomagnetically induced currents in Norway: the northernmost high-voltage power grid in the world. J Space Weather Space Clim 4:A10, doi: 10.1051/swsc/2014007

Ngwira M, McKinnell LA, Cilliers PJ (2011) Geomagnetic activity indicators for geomagnetically induced current studies in South Africa. Adv Space Res 48:529-534

Pirjola R (2002) Review on the calculation of surface electric and magnetic fields and of geomagnetically induced currents in ground based technological systems. Surv Geophys 23:71-90, doi:10.1023/A:1014816009303

Pirjola R (2005) Effects of space weather on high-latitude ground systems. Adv Space Res 36:2231-2240, doi:10.1016/j.asr.2003.04.074

Pirjola R (2008a) Study of effects of changes of earthing resistances on geomagnetically induced currents in an electric power transmission system. Radio Sci 43, RS1004, doi:10.1029/2007RS003704

Pirjola R (2008b) Effects of interactions between stations on the calculation of geomagnetically induced currents in an electric power transmission system. Earth Planets Space 60:743-751 
Pirjola R (2009) Properties of matrices included in the calculation of geomagnetically induced currents (GICs) in power systems and introduction of a test model for GIC computation algorithms. Earth Planets Space $61: 263-272$

Pirjola RJ (2010) On the flow of geomagnetically induced currents in an electric power transmission network. Can J Phys 88:357-363, doi:10.1139/P10-028

Pirjola R (2013) Practical model applicable to investigating the coast effect on the geoelectric field in connection with studies of geomagnetically induced currents. Adv Appl Phys 1:9-28

Pirjola RJ, Häkkinen LVT (1991) Electromagnetic field caused by an auroral electrojet current system model. In: Kikuchi $\mathrm{H}$ (ed) Environmental and space electromagnetics, Chapter 6.5. Springer, Tokyo, Japan, pp 288-298

Pirjola RJ, Viljanen AT (1991) Geomagnetic induction in the Finnish 400-kV power system. In: Kikuchi H (ed) Environmental and space electromagnetics, Chapter 6.4. Springer, Tokyo, Japan, pp 276-287

Pirjola R, Boteler D, Viljanen A, Amm O (2000) Prediction of geomagnetically induced currents in power transmission systems. Adv Space Res 26:5-14

Pous J, Ledo J, Marcuello A, Daignières M (1995) Electrical resistivity model of the crust and upper mantle from a magnetotelluric survey through the central Pyrenees. Geophys J Int 121:750-762

Pulkkinen A (2003) Geomagnetic induction during highly disturbed space weather conditions: studies of ground effects. Finnish Meteorological Institute Contributions, No. 42, Helsinki, Finland

Pulkkinen A, Amm O, Viljanen A, and the BEAR Working Group (2003) Ionospheric equivalent current distributions determined with the method of spherical elementary current systems. J Geophys Res 108:1053, doi:10.1029/2001JA005085

Pulkkinen A, Lindahl S, Viljanen A, Pirjola R (2005) Geomagnetic storm of 29-31 October 2003: geomagnetically induced currents and their relation to problems in the Swedish high-voltage power transmission system, Space Weather 3:S08C03., doi:10.1029/2004SW000123

Püthe C, Kuvshinov A (2013) Towards quantitative assessment of the hazard from space weather. Global 3-D modellings of the electric field induced by a realistic geomagnetic storm. Earth Planets Space 65:1017-1025, doi:10.5047/eps.2013.03.003

Royal Academy of Engineering (2013) Extreme space weather: impacts on engineered systems and infrastructure., http://www.raeng.org.uk/news/ publications/list/reports/space weather full report final.pdf. Accessed 8 April 2014

Song P, Singer HJ, Siscoe GL (2001) Space weather. AGU Geophysical Monograph 125. American Geophysical Union, Washington, D.C

Stolle C, Floberghagen R, Luhr H, Maus S, Knudsen DJ, Alken P, Doornbos E, Hamilton B, Thomson AWP, Visser PN (2013) Space weather opportunities from the Swarm mission including near real time applications. Earth Planets Space 65:1375-1383, doi:10.5047/eps.2013.10.002

Thomson AWP, McKay AJ, Clarke E, Reay S (2005) Surface electric fields and geomagnetically induced currents in the Scottish Power grid during the 30 October 2003 geomagnetic storm. Space Weather 3, S11002, doi:10.1029/2005SW000156

Thomson A, Reay S, Dawson E (2011) Quantifying extreme behaviour in geomagnetic activity. Space Weather 9, S10001, doi:10.1029/2011SW000696

Torta JM, Serrano L, Regué JR, Sánchez AM, Roldán E (2012) Geomagnetically induced currents in a power grid of northeastern Spain. Space Weather 10, S06002, doi:10.1029/2012SW000793

Trichtchenko L, Boteler DH (2002) Modelling of geomagnetic induction in pipelines. Ann Geophys 20:1063-1072

U.S. National Research Council (2008) Severe space weather eventsunderstanding societal and economic impacts. Committee on the Societal and Economic Impacts of Severe Space Weather Events: A Workshop. Space Studies Board. National Research Council. National Academies Press, Washington, D.C

Viljanen A (1998) Relation of geomagnetically induced currents and local geomagnetic variations. IEEE Trans Power Deliv 13:1285-1290

Viljanen A, Pirjola R (1989) Statistics on geomagnetically-induced currents in the Finnish $400 \mathrm{kV}$ power system based on recordings of geomagnetic variations. J Geomag Geoelectr 41:411-420

Viljanen A, Pirjola R (1994) Geomagnetically induced currents in the Finnish high-voltage power system. Surv Geophys 15:383-408

Viljanen A, Pulkkinen A, Pirjola R, Pajunpää K, Posio P, Koistinen A (2006) Recordings of geomagnetically induced currents and a nowcasting service of the Finnish natural gas pipeline system. Space Weather 4, S10004, doi:10.1029/2006SW000234

Viljanen A, Pirjola R, Wik M, Ádám A, Prácser E, Sakharov Y, Katkalov J (2012) Continental scale modelling of geomagnetically induced currents. J Space Weather Space Clim 2:A17, doi:10.1051/swsc/2012017

Wait JR (1981) Wave Propagation Theory. Pergamon, Tarrytown, New York Wik M, Viljanen A, Pirjola R, Pulkkinen A, Wintoft P, Lundstedt H (2008) Calculation of geomagnetically induced currents in the $400 \mathrm{kV}$ power grid in southern Sweden. Space Weather 6, S07005, doi:10.1029/2007SW000343

Wik M, Pirjola R, Lundstedt H, Viljanen A, Wintoft P, Pulkkinen A (2009) Space weather events in July 1982 and October 2003 and the effects of geomagnetically induced currents on Swedish technical systems. Ann Geophys 27:1775-1787

doi:10.1186/1880-5981-66-87

Cite this article as: Torta et al:: Assessing the hazard from geomagnetically induced currents to the entire high-voltage power network in Spain. Earth, Planets and Space 2014 66:87.

\section{Submit your manuscript to a SpringerOpen ${ }^{\odot}$ journal and benefit from:}

- Convenient online submission

- Rigorous peer review

- Immediate publication on acceptance

- Open access: articles freely available online

- High visibility within the field

- Retaining the copyright to your article

Submit your next manuscript at springeropen.com 